\title{
Wireless Three-hop Relay Environment with Line-of-Sight: Investigation and Performance Analysis
}

\author{
Dragana Krstic, Petar Nikolic, Zoran Popovic, Sinisa Minic, and Mihajlo Stefanovic
}

\begin{abstract}
Lately, the investigation of multi-hop relays is increased in both, academia and engineering practice. Multi-hop relay is introduced to enable data transmission between base station and mobile user dividing great distance into two or more segments to improve link quality. This improvement is specially expressed in environments under deep fading and shadow, where coverage is significantly increased using multi-hop relays. Here, wireless three-hop relay environment with line-of-sight was observed. Output signal from such system is defined as a product of three arbitrary, independent, but not necessarily identically distributed Rician random variables (RVs). For such system, some important performance of the first and second order were analyzed and graphically presented. The impact of Rician factor and signal powers on performance quantities was shown. Derived and displayed first order performance are: probability density function (PDF), cumulative distribution function (CDF), outage probability (Pout), moments, amount of fading (AoF) and channel capacity. Then, the following second order characteristics of wireless three-hop relay communication system working in Rician multipath fading environment were covered: level crossing rate (LCR) and average fade duration (AFD). These results have big application in wireless relay communications with a pronounced line-of-sight, where Rician model is used to describe fading.
\end{abstract}

Index Terms-Performance analysis, Rician fading, Three-hop relay system.

\section{INTRODUCTION}

$\mathrm{R}$ ELAYING is a promising improvement of current radio technologies. It is expected that relay technologies have

Manuscript received January 18, 2021; revised March 3, 2021. Date of publication August 24, 2021. Date of current version August 24, 2021. The associate editor prof. Andrej Hrovat has been coordinating the review of this manuscript and approved it for publication.

This paper is partially supported by projects: III-44006 and TR-33035 of the Serbian Ministry of Education, Science and Technological Development.

D. S. Krstic is with the Faculty of Electronic Engineering, University of Niš, Aleksandra Medvedeva 14, 18000 Nis, Serbia (e-mail: dragana.krstic@elfak.ni.ac.rs). P. B. Nikolić is with the Tigar Tyres, 18300 Pirot, Serbia (e-mail: nikpetar@gmail.com). Z. J. Popovic is with the Higher Technical School of Professional Studies, Zvecan, Serbia (e-mail: z.popovix@live.com). S. G. Minic is with the Teachers' Training Faculty, Prizren- Leposavic, University of Pristina, with headquarters in Kosovska Mitrovica, Serbia, (e-mail: sinisa.minic@pr.ac.rs). M. C. Stefanovic was with the Faculty of Electronic Engineering, University of Niš, Serbia, (e-mail: mihajlo.stefanovic@elfak.ni.ac.rs).

Digital Object Identifier (DOI): 10.24138/jcomss-2021-0013 higher cell coverage and capacity over the next generation wireless broadband radio access networks on an economical way. The organizations for standards development makes efforts to incorporate relay technologies into new standards. As these systems are newer, many open issues should be solved [1].

The conventional networks mostly use single-hop technology, where the end devices are connected through a direct link. There are different relaying architectures. Between them are multi-hop relays. They can provide additional capacity when fixed relays are inaccessible or insufficient. Using of multi-hop topologies in smart city applications enables energyefficient connections [2]-[6].

Because of that, it is interesting to investigate multi-hop, especially two-hop and three-hop, topologies by evaluating their performance [7]-[12]. Here, performance of the first and second order of wireless three-hop relay environment with lineof-sight will be considered. Observed first order performance are: probability density function (PDF), cumulative distribution function (CDF), outage probability (Pout), moments, amount of fading (AoF), channel capacity (CC), average symbol error probability (ASEP) or average bit error probability (ABEP) [4], [5], [10]- [13]. The level crossing rate (LCR) and the average outage duration (AOD) or average fade duration (AFD) are essential second order characteristics serving for deep analysis of the behavior of the fading environment [3], [14].

Radio signals arrive in antennas by two or more paths what causing significant variations of received signal amplitude and phase over time or space. This occurrence is multipath fading [15]. The short-term fading of the received envelope in can be described by dint of Rayleigh, Rician, Nakagami- $m$, Weibull, Hoyt [16], [17], and other more general distributions [18]-[24]. Rayleigh distribution is usually used for modeling signal amplitude in urban areas; Rician distribution is suitable for suburban areas which contain the line-of-sight (LOS) component; further, Weibull distribution shows good matching with to measured data from fading environment, while Nakagami- $m$ distribution shows exellent stacking in wide range of fading environments, what is proven experimentally as well as for indoor and outdoor radio channels [17]. 
This paper is organized as follows. After Introduction, in the section II the related papers are enumerated and system model presented. In the section III, the first order performance of wireless three-hop relay environment with line-of-sight are performed: PDF, CDF, Pout, moments, AoF, CC. The second order performance of this configuration, LCR and AFD, are illustrated in the section IV. For the product of three Rician RVs the graphs for all performance are presented to emphasize the parameter influence. The section $\mathrm{V}$ highlights possible applications of performed performance. The conclusion closes this work in section VI, and final are acknowledgment and list of references, as it is usual.

\section{RELATED PAPERS AND SYSTEM MODEL}

The Pout, CC and ASEP are derived for amplify-and-forward multihop relay network in the presence of Rayleigh fading in [5]. The second order statistical parameters, namely LCR and AFD of the amplify-and-forward multihop Rayleigh fading channel are presented in [3]. The channel is modelled as a product of $N$ fading amplitudes. Such case where Nakagami- $m$ fading is present in the channel is analyzed in [4], where some expressions for Pout are derived.

The amount of fading, as a simple measure for showing the performance of a diversity system, is discussed in [25]. In this paper, an approximations and bounds for AoF are provided. Also, a manner for derivation of the exact AoF calculations for transmit antenna selection on Rayleigh fading channels in Multiple Input Multiple Output (MIMO) systems is presented. The paper [26] analyzes an environment with independent and identically distributed (i.i.d.) double fading wich includes lineof-sight (LOS), typically presents in keyhole MIMO systems.

The considerations of three-hop relay systems is not represented so much in available literature. One of them is done in [21], where a product of three arbitrary, independent and n.i.d. $\alpha-\mu$ random variables (RVs) is observed. Closed-form expressions for PDF, CDF, moments, AoP and Pout are derived. The results are useful for MIMO links, multi-hop systems, radar communications, and cascaded communications.

Our group of authors considered first and second order performance of wireless three-hop relay channel under the influence of Rayleigh fading in [8]. The performance investigation of the output signal from a three-hop wireless relay system in the presence of Nakagami- $m$ fading, presented as a product of three Nakagami- $m$ RVs, is given in [9].

After analysis of non LOS fading conditions, modeled by Rayleigh and Nakagami distributions for three-hop wireless relay systems, we consider such scenario with Rician distribution for fading, which models LOS environment. First, in [10], PDF and first two moments are obtained. AoF is performed based on these moments. The LCR is derived in [11]. In [12], CDF and Pout are determined. Further, based on previously determined LCR and Pout, AFD is obtained and graphicaly shown. In this paper, the third moment will supplement derivation of moments, and channel capacity will be found.
The CC per unit bandwidth for two different adjustment policies over $\kappa-\mu$ fading channels with maximal ratio combinig (MRC) diversity were considered in [27]. Channel capacity of the macrodiversity selection combinig (SC) receiver consisting of two dual branch SC microdiversity receivers, in the presence of $\kappa-\mu$ fading and correlated slow gamma fading is determined in [28].

Channel capacity of MRC over exponentially correlated Nakagami- $m$ fading channels for optimal power and rate adaptation policy is discussed in [29]. Later, capacity of dual branch MRC system over correlated Nakagami- $m$ fading channels is presented as a review in [13]. Also, uncorrelated Nakagami- $m$ fading channels are observed as well for $m=1$, which is special case of Rayleigh fading channel.

Knowing of the second-order performance of fading environment (LCR AFD) helps to understand and mitigate the impact of fading. AFD determines the average length of error bursts in fading environment. So, if AFD is relatively large, long data blocks will be significantly affected by the channel fades than short blocks. Also, a knowledge of these performance is important for many applications such as: correct choice of the frame length for coded packetized systems, design of (non)interleaved coding methods, optimal size of interleaver, estimation of communication protocols, choosing the buffer depth for adaptive modulation schemes.

With fast increasing of wireless communication, there is an extensive growth in the demand of radio frequency through electromagnetic spectrum allocation. In this regard, cognitive radio is a potential technology helping to overcome the request in radio frequency. In [30], relaying protocol over a Weibull fading channels in cognitive radio is used to improve the efficiency and to minimize the bit error rate during transmission. The numerical results showed that multi-hop has better performance compared to single-hop relaying protocol.

It is known that performance of free-space optics (FSO) links are very depending on scintillation effects caused by the atmospheric turbulences. An accepted approach to counterbalance the expected spoilage due to scintillation is to use multi-hop relays. This concept greatly increases the mathematical complexity of the models for describing the system performance. Because of that, the most often is used a dual-hop link in order to simplify the performance analysis. In [31], mathematical derivations for the evaluation of the outage probability and the average bit error rate were made by assuming typical turbulence models.

Although the mathematical complexity of the models for describing $N$-hop relays increases with growth of $N$, and many authors choose to analyze dual-hop relays, we tackled with derivation in closed forms some performance of three-hop relays here. Such a three-hop relay system is illustrated in Fig. 1 [6]. The system consists of the source who sends the information to the destination using two consecutive relays, which can be of AF or DF type. The relays help reliable transmission of the information to the destination. The relays are necessary when network nodes suffer heavy shadowing; distance between terminals is big, and also for the case with limited power resources [32]. 


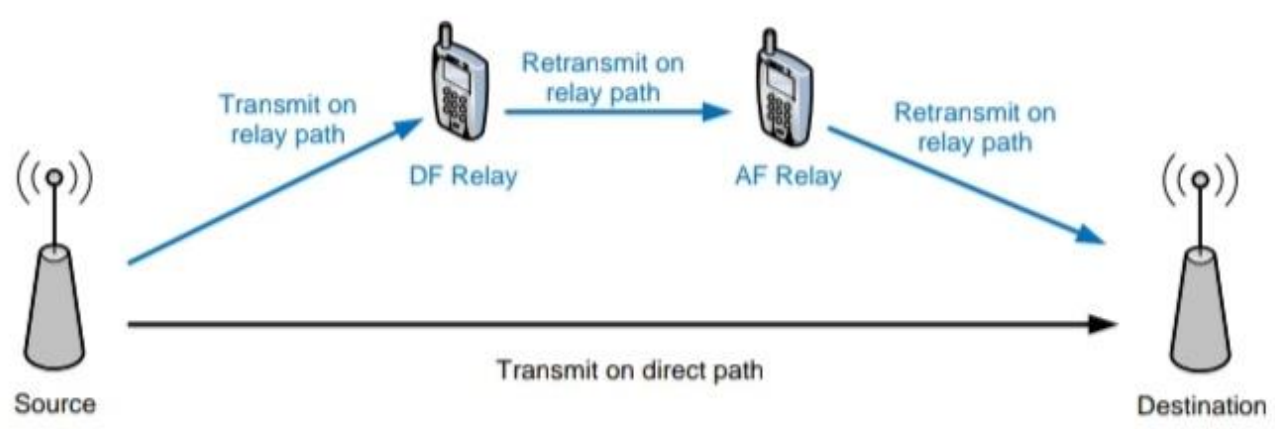

Fig. 1. Three-hop relay system [6].

\section{THE FIRST ORDER PERFORMANCE OF WIRELESS THREE- HOP RELAY ENVIRONMENT WITH LINE-OF-SIGHT}

For analysis of three-hop wireless relay system from Fig. 1, it is necessary to determine the first-order performance of the product of three Rician random variables.

\section{A. PDF of Product of Three Rician Random Variables}

In mobile communication systems, when a Line of Sight (LoS) component exists between the transmitter and the receiver, the received signal can be presented as the sum of a complex exponential and a narrowband Gaussian process. The first one is known as the LoS component and the other is diffuse component. The ratio of the powers of the LoS component to the diffuse component is the $\kappa$ factor, which measures the relative strength of the LoS component and shows a measure of link quality [33].

The most important role in the performance of the wireless systems has the communication link quality. The link quality knowledge gives the possibility to the system to characterize the channel and accommodate to the current transmission conditions.

In the observed case, the time varying envelopes $x_{i}$ of the received signals are present in all three hops and hence described by a Rician distribution given by [34]:

$$
\begin{array}{r}
p_{x_{i}}\left(x_{i}\right)=\frac{2\left(\kappa_{i}+1\right)}{\Omega_{\mathrm{i}} \mathrm{e}^{\kappa_{i}}} \sum_{j_{i}=0}^{\infty}\left(\frac{\left(\kappa_{i}+1\right) \kappa_{i}}{\Omega_{i}}\right)^{j_{i}} \frac{1}{\left(j_{i} !\right)^{2}} . \\
\cdot x_{i}^{2 j_{i}+1} e^{-\frac{\kappa_{i}+1}{\Omega_{\mathrm{i}}} x_{i}^{2}}, x_{i} \geq 0,
\end{array}
$$

where $\kappa_{\mathrm{i}}$ are parameters of Rician distribution at $i$-th hop, called Rician factors of RVs $x_{\mathrm{i}}$ and $\Omega_{\mathrm{i}}$ are mean powers of RVs $x_{\mathrm{i}}$. Therefore, Rician factor is defined as a ratio of the signal power of dominant component of the signal and powers of the scattered components and represents a measure of the severity of the fading. When Rician factor $\kappa \rightarrow 0$, the distribution becomes Rayleigh distribution and this is most severe Rayleigh fading. The case when $\kappa \rightarrow \infty$ indicates a case that there is no fading in a propagation channel. $\kappa$ factor estimation is critical for many wireless applications, as link budget calculations, precoding, adaptive modulation, and others [35].
$\mathrm{RV} x$ is defined as the product of three independent Rician RVs: $x=x_{1} \cdot x_{2} \cdot x_{3}$.

PDF of product of three Rician RVs, obtained in [10, eq. (7)], is presented in Appendix as (A6):

$$
\begin{gathered}
p_{x}(x)=\frac{2\left(\kappa_{1}+1\right)}{\Omega_{1} \mathrm{e}^{\kappa_{1}}} \sum_{j_{1}=0}^{\infty}\left(\frac{\left(\kappa_{1}+1\right) \kappa_{1}}{\Omega_{1}}\right)^{j_{1}} \frac{1}{\left(j_{1} !\right)^{2}} . \\
\frac{2\left(\kappa_{2}+1\right)}{\Omega_{2} \mathrm{e}^{\kappa_{2}}} \sum_{j_{2}=0}^{\infty}\left(\frac{\left(\kappa_{2}+1\right) \kappa_{2}}{\Omega_{2}}\right)^{j_{2}} \frac{1}{\left(j_{2} !\right)^{2}} . \\
\frac{2\left(\kappa_{3}+1\right)}{\Omega_{3} \mathrm{e}^{\kappa_{3}}} \sum_{j_{1}=0}^{\infty}\left(\frac{\left(\kappa_{3}+1\right) \kappa_{3}}{\Omega_{3}}\right)^{j_{1}} \frac{1}{\left(j_{3} !\right)^{2}} . \\
\int_{0}^{\infty} d x_{2} \int_{0}^{\infty} d x_{3} x_{2}^{-1-2 j_{1}+2 j_{2}} x_{3}^{-1-2 j_{1}+2 j_{3}} \\
\cdot x^{2 j_{1}+1} e^{-\frac{\kappa_{1}+1}{\Omega_{1}}\left(\frac{x}{x_{2} x_{3}}\right)^{2}-\frac{\kappa_{2}+1}{\Omega_{2}} x_{2}^{2}-\frac{\kappa_{3}+1}{\Omega_{3}} x_{3}^{2}} .
\end{gathered}
$$

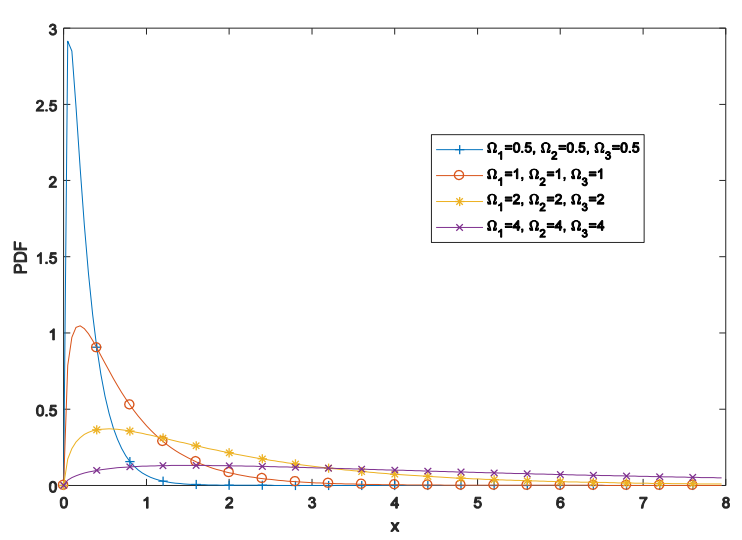

Fig. 2. PDF of product of three Rician RVs for different values of the signal powers for $\kappa_{1}=\kappa_{2}=\kappa_{3}=1[10]$.

In Fig. 2, PDF of product of three Rician RVs is graphically presented versus this product $x$ for different values of signal average powers $\Omega_{i}$ at relay sections (hops) and Rician factors 
$\kappa_{\mathrm{i}}=1, i=1,2,3$ by using mathematical software Matlab. One can see from this picture that PDF increases for low $x$, reaches maximum and then start to decrease for bigger values of the product $x$ for all values of the signal powers $\Omega_{i}$. It is noticeable that small values of $x$ affect more to the PDF.

\section{B. CDF of Product of Three Rician Random Variables}

Cumulative distribution function (CDF) of product of three Rician RVs is obtained in [12, Eq. (4)]:

$$
\begin{aligned}
& F_{x}(x)=\int_{0}^{\infty} d t p_{x}(t)= \\
& =\frac{2\left(\kappa_{1}+1\right)}{\Omega_{1} \mathrm{e}^{\kappa_{1}}} \sum_{j_{1}=0}^{\infty}\left(\frac{\left(\kappa_{1}+1\right) \kappa_{1}}{\Omega_{1}}\right)^{j_{1}} \frac{1}{\left(j_{1} !\right)^{2}} . \\
& \cdot \frac{2\left(\kappa_{2}+1\right)}{\Omega_{2} \mathrm{e}^{\kappa_{2}}} \sum_{j_{2}=0}^{\infty}\left(\frac{\left(\kappa_{2}+1\right) \kappa_{2}}{\Omega_{2}}\right)^{j_{2}} \frac{1}{\left(j_{2} !\right)^{2}} . \\
& \cdot \frac{2\left(\kappa_{3}+1\right)}{\Omega_{3} \mathrm{e}^{\kappa_{3}}} \sum_{j_{1}=0}^{\infty}\left(\frac{\left(\kappa_{3}+1\right) \kappa_{3}}{\Omega_{3}}\right)^{j_{1}} \frac{1}{\left(j_{3} !\right)^{2}} . \\
& \int_{0}^{\infty} d x_{2} \int_{0}^{\infty} d x_{3} x_{2}^{-1-2 j_{1}+2 j_{2}+2 j_{1}+2} x_{3}^{-1-2 j_{1}+2 j_{3}+2 j_{1}+2} \\
& \cdot e^{-\frac{\kappa_{2}+1}{\Omega_{2}} x_{2}^{2}-\frac{\kappa_{3}+1}{\Omega_{3}} x_{3}^{2}} \\
& \cdot \frac{1}{2}\left(\frac{\Omega_{1}}{\kappa_{1}+1}\right)^{j_{1}+1} \gamma\left(j_{1}+1, \frac{\kappa_{1}+1}{\Omega_{1}} \frac{x^{2}}{x_{2}^{2} x_{3}^{2}}\right) \text {. }
\end{aligned}
$$

\section{Outage probability of Product of Three Rician Random Variables}

The outage probability is an important performance measure of communication systems working in fading environment. Pout is defined as the probability that information rate is below the predifined threshold $\Gamma_{\text {th }}$, actually, Pout is the probability that an outage is occurred within a given time period:

$$
P_{\text {out }}=\int_{0}^{\Gamma_{\text {th }}} p_{x}(t) d t \text {. }
$$

Here, $p_{x}(x)$ is the signal PDF and $\Gamma_{\text {th }}$ is the system protection ratio which depend on the type of used modulation and the characteristics of receiver [36].

When (2) is put in (3), Pout becomes:

$$
P_{\text {out }}=F_{x}\left(\Gamma_{t h}\right) .
$$

Some graphs for Pout for this system are shown in Figs. 3 and 4 for some parameters values [12]. It is obvious that performance is improved with increasing of Rician factors $\kappa_{\mathrm{i}}$. Besides, bigger values of fading powers $\Omega_{\mathrm{i}}$ reduce the Pout and improve system performance.

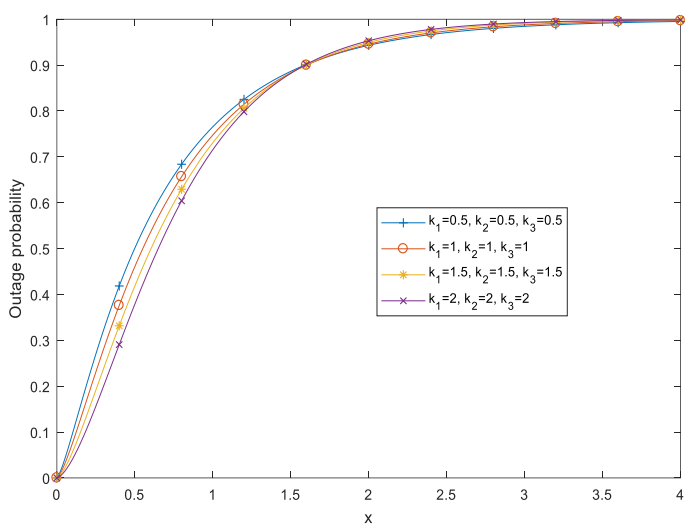

Fig. 3. Outage probability of product of three Rician RVs versus signal envelope $x$ for different values of Rician factor $\kappa_{i}$ and signal power $\Omega_{\mathrm{i}}=1$ [12].

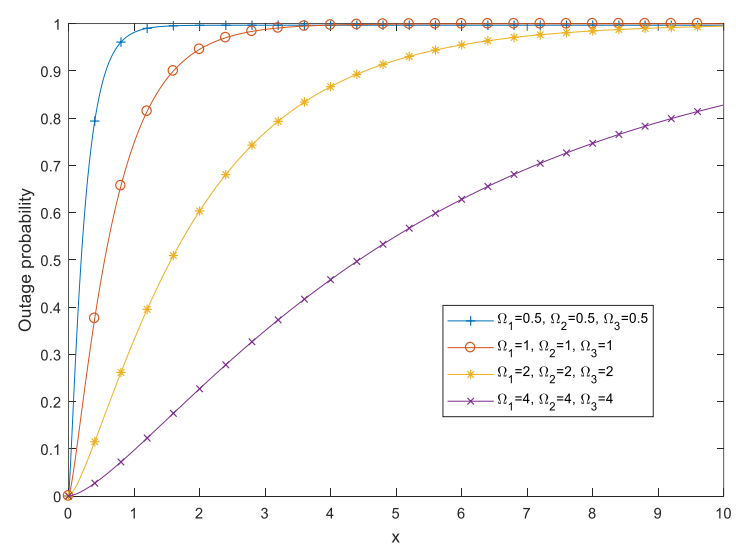

Fig. 4. Pout of product of three Rician RVs depending on signal envelope for various values of signal power $\Omega_{\mathrm{i}}$ and Rician factor $\kappa_{\mathrm{i}}=1$ [12].

\section{Moments of Product of Three Rician Random Variables}

Usually, the PDF of a variable gives its complete picture, but sometimes, only a partial description is necessary or possible. This is provided by statistical average values. They play an essential role in the description of the RV. These statistical average values are, in fact, moments. For statistical analysis the most interesting are the first few moments of RV and joint moments (the correlation and the covariance), between any pair of RVs in a multidimensional set of RVs [37].

The expected value, also known as the mean value of the product of three Rician RVs $x$ is defined by the formula [37]:

$$
m_{x}=E[x]=\int_{0}^{\infty} x p(x) d x .
$$

Here, $E$ is the statistical expectation operator. Since $p_{x}(x) d x$ is the probability of RV $x$ being in the infinitesimal strip $d x, m_{x}$ is interpreted as the weighted average of $x$. So, each weight is the probability of occurrence of the specific value $x$. This is called the first moment of a RV. For our product of three RVs it is given and solved in closed form in [10, Eq. (9)]:

$$
m_{1}=\bar{x}=\frac{2\left(\kappa_{1}+1\right)}{\Omega_{1} \mathrm{e}^{\kappa_{1}}} \sum_{j_{1}=0}^{\infty}\left(\frac{\left(\kappa_{1}+1\right) \kappa_{1}}{\Omega_{1}}\right)^{j_{1}} \frac{1}{\left(j_{1} !\right)^{2}} .
$$




$$
\begin{gathered}
\cdot \frac{2\left(\kappa_{2}+1\right)}{\Omega_{2} \mathrm{e}^{\kappa_{2}}} \sum_{j_{2}=0}^{\infty}\left(\frac{\left(\kappa_{2}+1\right) \kappa_{2}}{\Omega_{2}}\right)^{j_{2}} \frac{1}{\left(j_{2} !\right)^{2}} . \\
\cdot \frac{2\left(\kappa_{3}+1\right)}{\Omega_{3} \mathrm{e}^{\kappa_{3}}} \sum_{j_{1}=0}^{\infty}\left(\frac{\left(\kappa_{3}+1\right) \kappa_{3}}{\Omega_{3}}\right)^{j_{1}} \frac{1}{\left(j_{3} !\right)^{2}} \cdot \\
\cdot \frac{1}{2}\left(\frac{\Omega_{1}}{\kappa_{1}+1}\right)^{j_{1}+3 / 2} \Gamma\left(j_{1}+3 / 2\right) \cdot \frac{1}{2}\left(\frac{\Omega_{2}}{\kappa_{2}+1}\right)^{j_{2}+3 / 2} \Gamma\left(j_{2}+3 / 2\right) \\
\cdot \frac{1}{2}\left(\frac{\Omega_{3}}{\kappa_{3}+1}\right)^{j_{3}+3 / 2} \Gamma\left(j_{3}+3 / 2\right) .
\end{gathered}
$$

The first moment for the product of three Rician RVs is graphically presented in Fig. 5 [10]. One can see from Fig. 5 that the first moment is higher for higher values of $\Omega_{i}$. Also, the first moment, $m_{l}$, increases with increasing of $\kappa$ till maximum, when $m_{l}$ start to decline.

The second moment is also very important. It is known as the mean-squared value or variance or the signal's average power of the RV and is determined in [10] for the product of three Rician RVs as:

$$
\begin{gathered}
m_{2}=\overline{x^{2}}=\int_{0}^{\infty} d x x^{2} p(x)= \\
=\frac{2\left(\kappa_{1}+1\right)}{\Omega_{1} \mathrm{e}^{\kappa_{1}}} \sum_{j_{1}=0}^{\infty}\left(\frac{\left(\kappa_{1}+1\right) \kappa_{1}}{\Omega_{1}}\right)^{j_{1}} \frac{1}{\left(j_{1} !\right)^{2}} \cdot \\
\cdot \frac{2\left(\kappa_{2}+1\right)}{\Omega_{2} \mathrm{e}^{\kappa_{2}}} \sum_{j_{2}=0}^{\infty}\left(\frac{\left(\kappa_{2}+1\right) \kappa_{2}}{\Omega_{2}}\right)^{j_{2}} \frac{1}{\left(j_{2} !\right)^{2}} \cdot \\
\cdot \frac{2\left(\kappa_{3}+1\right)}{\Omega_{3} \mathrm{e}^{\kappa_{3}}} \sum_{j_{1}=0}^{\infty}\left(\frac{\left(\kappa_{3}+1\right) \kappa_{3}}{\Omega_{3}}\right)^{j_{1}} \frac{1}{\left(j_{3} !\right)^{2}} \cdot \\
\cdot \frac{1}{2}\left(\frac{\Omega_{1}}{\kappa_{1}+1}\right)^{j_{1}+2} \Gamma\left(j_{1}+2\right) \cdot \frac{1}{2}\left(\frac{\Omega_{2}}{\kappa_{2}+1}\right)^{j_{2}+2} \Gamma\left(j_{2}+2\right) \\
\cdot \frac{1}{2}\left(\frac{\Omega_{3}}{\kappa_{3}+1}\right)^{j_{3}+2} \Gamma\left(j_{3}+2\right) \cdot
\end{gathered}
$$

The positive square root of the variance is the standard deviation. When speaking about wireless communication, we are talking about signal's average power. The parameters influence to the second moment of product of three Rician RVs is shown here in Figs. 6 and 7 [10].

The second moment, or variance, grows with increasing of power $\Omega$. For bigger $\Omega$ and Rician factor $\kappa, m_{2}$ is declining.

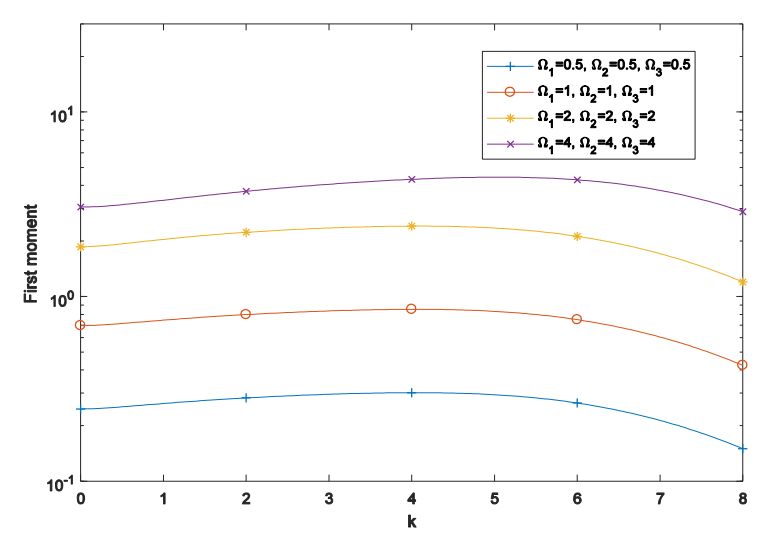

Fig. 5. Dependence of first moment of the product of three Rician RVs on Rician factor $\kappa$ for a few values of signal powers $\Omega_{\mathrm{i}}[10]$.

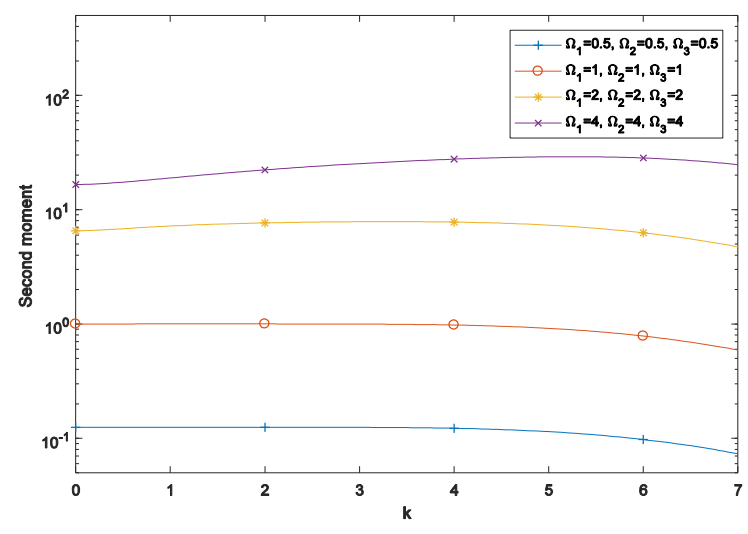

Fig. 6. The second moment of product of three Rician RVs depending on Rician factor $\kappa$ for variable signal power $\Omega_{\mathrm{i}}[10]$.

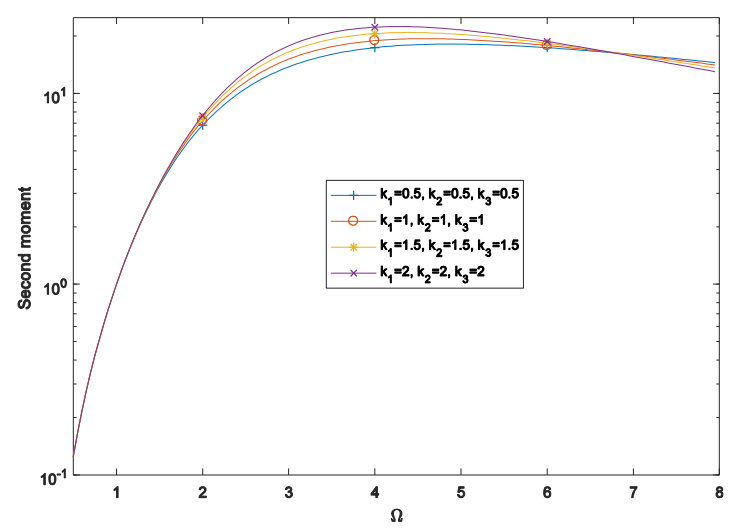

Fig. 7. The second moment of product of three Rician RVs depending on signal power $\Omega$ for different values of Rician factor $\kappa_{i}[10]$.

The next considered moment is the normalized third central moment, called the skewness. It is a measure of limitedness of the distribution or the asymmetry of the probability distribution of a real-valued RV about its mean value.

The skewness can have positive or negative value, or even be undefined. The third moment for symmetric distribution, if is defined, is equal to zero. If the distribution is skewed to the left, it has negative value of skewness and vice versa, the distribution 
which is skewed to the right has positive value of skewness.

The third moment, $m_{3}$, is defined by the formula:

$$
m_{3}=\overline{x^{3}}=\int_{0}^{\infty} d x x^{3} p_{x}(x) .
$$

The final shape of this moment is performed as previous ones:

$$
\begin{gathered}
m_{3}=\frac{2\left(\kappa_{1}+1\right)}{\Omega_{1} \mathrm{e}^{\kappa_{1}}} \sum_{j_{1}=0}^{\infty}\left(\frac{\left(\kappa_{1}+1\right) \kappa_{1}}{\Omega_{1}}\right)^{j_{1}} \frac{1}{\left(j_{1} !\right)^{2}} . \\
\cdot \frac{2\left(\kappa_{2}+1\right)}{\Omega_{2} \mathrm{e}^{\kappa_{2}}} \sum_{j_{2}=0}^{\infty}\left(\frac{\left(\kappa_{2}+1\right) \kappa_{2}}{\Omega_{2}}\right)^{j_{2}} \frac{1}{\left(j_{2} !\right)^{2}} \cdot \\
\cdot \frac{2\left(\kappa_{3}+1\right)}{\Omega_{3} \mathrm{e}^{\kappa_{3}}} \sum_{j_{1}=0}^{\infty}\left(\frac{\left(\kappa_{3}+1\right) \kappa_{3}}{\Omega_{3}}\right)^{j_{1}} \frac{1}{\left(j_{3} !\right)^{2}} \cdot \\
\left.\cdot \frac{1}{2}\left(\frac{\Omega_{1}}{\kappa_{1}+1}\right)^{j_{1}+2}{ }^{j_{1}+2}+5 / 2\right) \cdot \frac{1}{2}\left(\frac{\Omega_{2}}{\kappa_{2}+1}\right)^{j_{2}}\left(j_{2}+5 / 2\right) \\
\cdot \frac{1}{2}\left(\frac{\Omega_{3}}{\kappa_{3}+1}\right)^{j_{3}+2} \Gamma\left(j_{3}+5 / 2\right) .
\end{gathered}
$$

Graphs for the third moment of product of three Rician RVs versus signal power $\Omega$ and Rician factor $\kappa$ are plotted in Figs. 8 and 9. It is visible that the third moment decreases slightly with higher values of the Rician factor $\kappa$.

From Fig. 9 is evident that third moment increases with increasing of $\Omega$, reaches a maximum and begins to slowly decline.

In Fig. 10, the first three moments of product of three Rician RVs, are shown versus signal power $\Omega$. All three moments increase with an increase of $\Omega$ and quickly reach saturation.

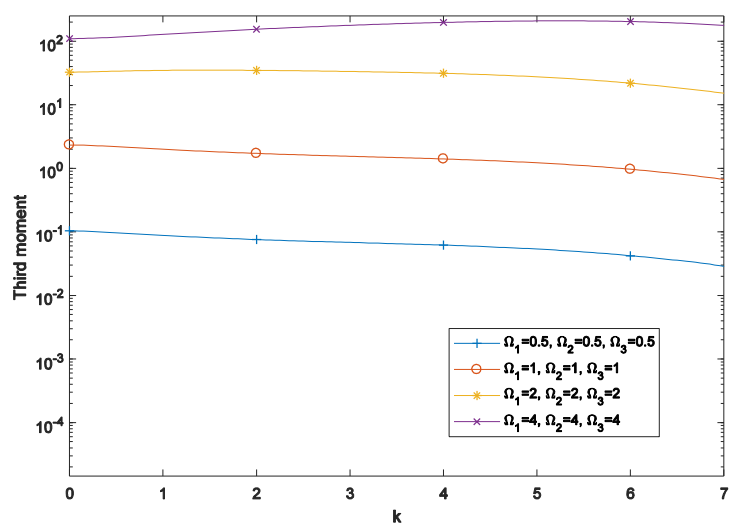

Fig. 8. The third moment of product of three Rician RVs versus Rician factor $\kappa$ for different values of the signal power $\Omega_{\mathrm{i}}$.

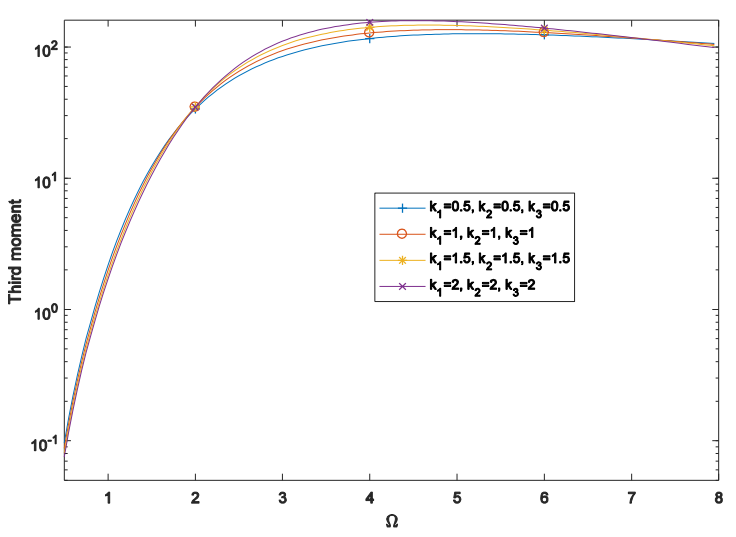

Fig. 9. The third moment of product of three Rician RVs depending on the signal power $\Omega$ with Rician factor $\kappa_{i}$ as the parameter of curves.

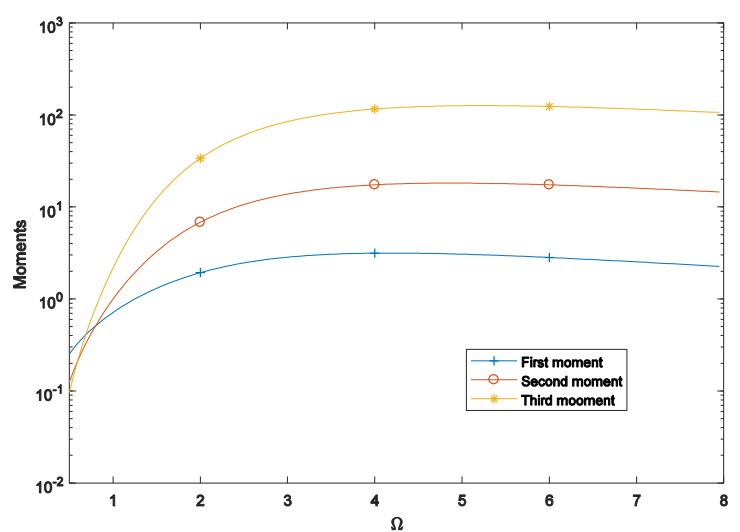

Fig. 10. The moments of product of three Rician RVs versus signal power $\Omega$.

General, $n$-th moment is defined by [37, Eq. (3.26)]. $n$-th moment of product of three Rician RVs, $x$, is derived in [10, Eq. (11)] as:

$$
\begin{gathered}
m_{n}=\overline{x^{n}}=\int_{0}^{\infty} d x x^{n} p(x)= \\
=\frac{2\left(\kappa_{1}+1\right)}{\Omega_{1} \mathrm{e}^{\kappa_{1}}} \sum_{j_{1}=0}^{\infty}\left(\frac{\left(\kappa_{1}+1\right) \kappa_{1}}{\Omega_{1}}\right)^{j_{1}} \frac{1}{\left(j_{1} !\right)^{2}} \cdot \\
\cdot \frac{2\left(\kappa_{2}+1\right)}{\Omega_{2} \mathrm{e}^{\kappa_{2}}} \sum_{j_{2}=0}^{\infty}\left(\frac{\left(\kappa_{2}+1\right) \kappa_{2}}{\Omega_{2}}\right)^{j_{2}} \frac{1}{\left(j_{2} !\right)^{2}} \cdot \\
\left.\left.\cdot \frac{2\left(\kappa_{3}+1\right)}{\Omega_{3} \mathrm{e}^{\kappa_{3}}} \sum_{j_{1}=0}^{\infty}\left(\frac{\left(\kappa_{3}+1\right) \kappa_{3}}{\Omega_{3}}\right)^{j_{1}} \frac{1}{\Omega_{1}}\right)^{j_{1}+2} \cdot j_{3}\right)^{2} \\
\Gamma\left(j_{1}+1+n / 2\right) \cdot \frac{1}{2}\left(\frac{\Omega_{2}}{\kappa_{2}+1}\right)^{j_{2}+2} \Gamma\left(j_{2}+1+n / 2\right) \\
\cdot \frac{1}{2}\left(\frac{\Omega_{3}}{\kappa_{3}+1}\right)^{j_{3}+2} \Gamma\left(j_{3}+1+n / 2\right) .
\end{gathered}
$$




\section{E. Amount of Fading of Product of Three Rician RVs}

The amount of fading is an indicator of severity of fading for observed environment. For a defined distribution of received signal power, the AoF is defined as a ratio of the variance of received energy to the square of the mean of received energy [38, Eq. (1)], [17, eq. (2.5)]:

$$
A o F=\operatorname{Var}\left\{x^{2}\right\} /\left(E\left\{x^{2}\right\}\right)^{2}
$$

where $\mathrm{E}\{\cdot\}$ and $\operatorname{Var}\{\cdot\}$ designate the statistical average and variance, in a row.

The moments of fading distribution are directly used for calculating the AoF. So, it is a simple manner to quantify fading [39, Eq. (9)]:

$$
A o F=m_{2} / m_{1}^{2}-1
$$

The AoF values are in the range [0,2]. For environment with line-of-sight, the fading channel is close to an additive white Gaussian noise (AWGN) channel.

If $\mathrm{AoF}=0$, this is "no fading" environment; $\mathrm{AoF}=1$ corresponds to a SISO Rayleigh fading channel; $\mathrm{AF}=1 / \mathrm{m}$ in a generalized Nakagami-m fading channel, and finally, $\mathrm{AoF}=2$ for one-sided Gaussian distribution, what is the most severe fading.

In Fig. 11, AoF of product of three Rician RVs is presented versus Rician factor $\kappa$ for some different values of $\Omega_{\mathrm{i}}$.

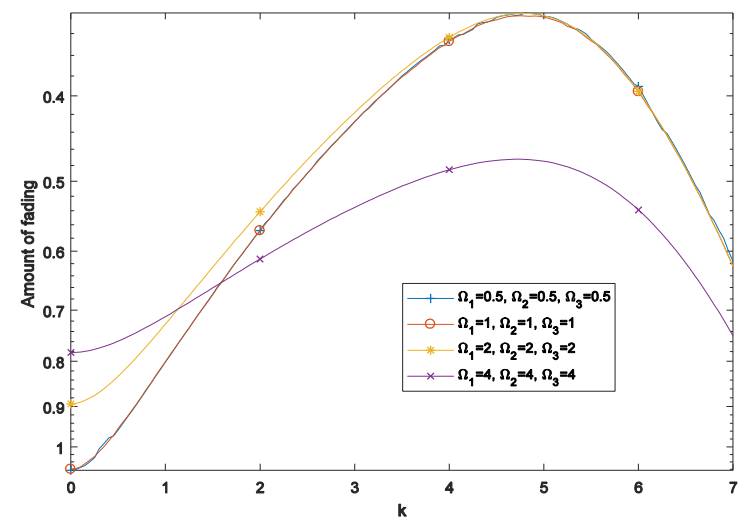

Fig. 11. The amount of fading of product of three Rician RVs versus Rician factor $\kappa$ for changeable signal powers $\Omega_{\mathrm{i}}[10]$.

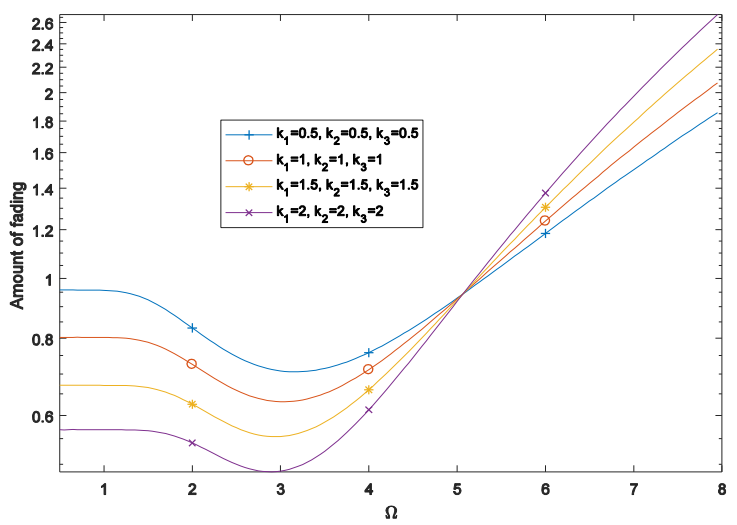

Fig. 12. AoF of product of three Rician RVs depending on signal power $\Omega$ for different values of Rician factors $\kappa_{i}[10]$
In the next, Fig. 12, AoF is plotted depending on mean powers $\Omega$ for a few values of Rician factors $\kappa_{\mathrm{i}}$. It is possible to notice from this graphs that AoF increases with increasing of $\Omega$ for bigger $\Omega$. For higher values of $\kappa_{\mathrm{i}}$ and small $\Omega$, AoF has low values, but the case is completely different for bigger $\Omega$. There, AoF has higher values for higher Rician factor $\kappa$.

\section{F. Channel Capacity of Product of Three Rician Random Variables}

In information theory, channel capacity (CC) is the upper bound on the rate at which information can be reliably transmitted over a communication channel. The $\mathrm{CC}$ of a given channel is the highest information rate that can be achieved with arbitrarily small error probability. It is expressed in units of information per unit time. The term of $\mathrm{CC}$ was key for the development of modern wireless communication systems and achieving performance close to the promised limits.

Assuming the transmitting power of the system is constant, and optimal rate adaptation (ORA) technique it has been applied at the transmitter, the channel capacity can be expressed as [40]:

$$
C=B \int_{0}^{\infty} \log _{2}(1+x) p_{x}(x) d x
$$

where $\mathrm{B}$ denotes channel bandwith expressed in $\mathrm{Hz}$.

When we have derived expression for the PDF, we can easy calculate the channel capacity at the output of the relay system. The maximum data rate can be reached after the chanel is given in the unit of bits per second.

By substituting expression (1) in (13), we obtain the normalized $\mathrm{CC}$ as:

$$
\begin{aligned}
& \frac{C}{B}=\int_{0}^{\infty} d x \log _{2}(1+x) \frac{2\left(\kappa_{1}+1\right)}{\Omega_{1} e^{\kappa_{1}}} \sum_{j_{1}=0}^{\infty}\left(\frac{\left(\kappa_{1}+1\right) \kappa_{1}}{\Omega_{1}}\right)^{j_{1}} \frac{1}{\left(j_{1} !\right)^{2}} . \\
& \cdot \frac{2\left(\kappa_{2}+1\right)}{\Omega_{2} \mathrm{e}^{\kappa_{2}}} \sum_{j_{2}=0}^{\infty}\left(\frac{\left(\kappa_{2}+1\right) \kappa_{2}}{\Omega_{2}}\right)^{j_{2}} \frac{1}{\left(j_{2} !\right)^{2}} . \\
& \frac{2\left(\kappa_{3}+1\right)}{\Omega_{3} \mathrm{e}^{\kappa_{3}}} \sum_{j_{1}=0}^{\infty}\left(\frac{\left(\kappa_{3}+1\right) \kappa_{3}}{\Omega_{3}}\right)^{j_{1}} \frac{1}{\left(j_{3} !\right)^{2}} . \\
& \int_{0}^{\infty} d x_{2} \int_{0}^{\infty} d x_{3} x_{2}^{-1-2 j_{1}+2 j_{2}} x_{3}^{-1-2 j_{1}+2 j_{3}} \\
& \cdot x^{2 j_{1}+1} e^{-\frac{\kappa_{1}+1}{\Omega_{1}}\left(\frac{x}{x_{2} x_{3}}\right)^{2}-\frac{\kappa_{2}+1}{\Omega_{2}} x_{2}^{2}-\frac{\kappa_{3}+1}{\Omega_{3}} x_{3}^{2}} .
\end{aligned}
$$

The channel capacity graphs are presented in Fig. 13 and 14 . The Fig. 13 gives dependence of $\mathrm{CC}$ from Rician factor $\kappa$. One can notice from this figure that $\mathrm{CC}$ increases for small versus of Rician factor $\kappa$. Then, $\mathrm{CC}$ acheives maximal value and starts to decrease, with a rapid approach to zero. 


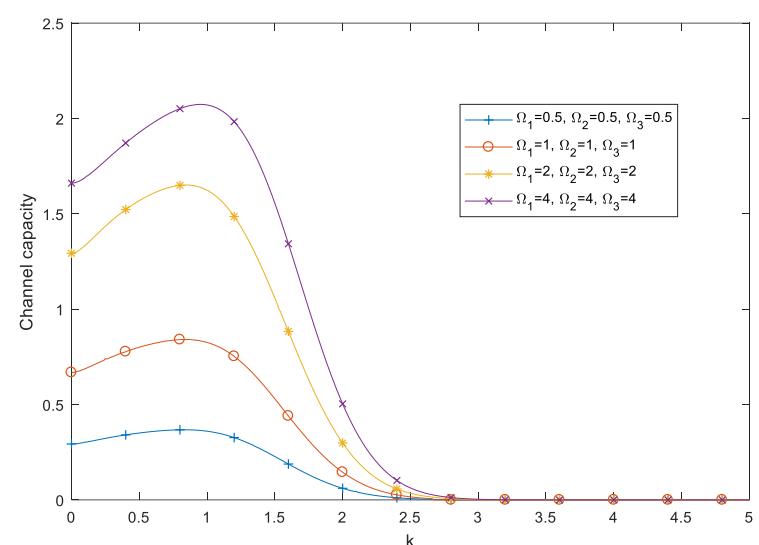

Fig. 13. Normalized channel capacity, C/B, of product of three Rician RVs versus Rician factor $\kappa$, where $\Omega$ changes.

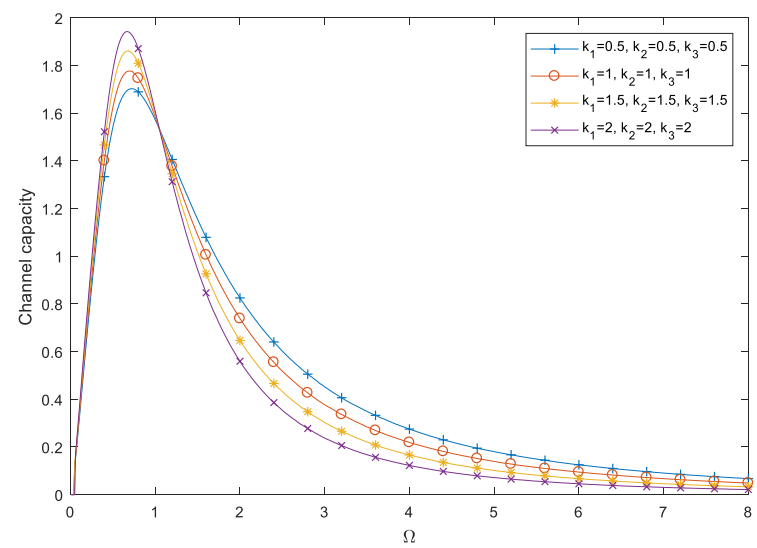

Fig. 14. C/B for product of three Rician RVs depending on power $\Omega$ and changeable Rician factor.

The Fig. 14 shows the CC versus signal power $\Omega$ for different values of Rician factor $\kappa_{\mathrm{i}}$. It can be spotted that low $x$ affect more to the $\mathrm{CC}$. For that values, $\mathrm{CC}$ grows fast, reaches maximum, and begins to decline.

\section{THE SECOND ORDER PERFORMANCE OF WIRELESS THREE-HOP RELAY ENVIRONMENT WITH LINE-OF-SIGHT}

Level crossing rate and average fade duration are very important features of the second-order for wireless channels. They provide useful data of the dynamic behavior of wireless fading environment.

\section{A. Level Crossing Rate of Product of Three Rician RVs}

LCR is one of the most important second-order performance measures of wireless communication systems. It is applicable in modelling and design of communication systems as well as throughput analysis, optimization of interleave size and designing of error correcting codes.

The LCR is defined as the expected rate at which a fading envelope crosses defined level in the downward, or updown, direction and is expressed in crossings per second [41]. The LCR of RV informs how often the envelope crosses a given threshold $x$ [42].

It is necessary to find an expression for the joint probability density function (JPDF) of $x$ and $\dot{x}$, signed $p_{x \dot{x}}(x \dot{x})$. Applaying the Rice's formula [34, Eq. (2.106)], the LCR can be calculated [16] as:

$$
N_{x}=\int_{0}^{\infty} d \dot{x} \dot{x} p_{x \dot{x}}(x \dot{x}) .
$$

LCR of output of three-hop relay system, actually of product of three Rician RVs is already derived in [11, eq. (20)]:

$$
\begin{gathered}
N_{x}=\frac{1}{\sqrt{2 \pi}} \pi f_{m} \frac{\Omega_{1}^{1 / 2}}{\left(\kappa_{1}+1\right)^{1 / 2}} \cdot \frac{2\left(\kappa_{1}+1\right)}{\Omega_{1}} \cdot \frac{2\left(\kappa_{2}+1\right)}{\Omega_{2}} \cdot \frac{2\left(\kappa_{3}+1\right)}{\Omega_{3}} . \\
\cdot \sum_{i_{1}=0}^{\infty} \sum_{i_{2}=0}^{\infty} \sum_{i_{3}=0}^{\infty}\left(\frac{\kappa_{1}\left(\kappa_{1}+1\right)}{\Omega_{1}}\right)^{i_{1}} \frac{1}{\left(i_{1} !\right)^{2}}\left(\frac{\kappa_{2}\left(\kappa_{2}+1\right)}{\Omega_{2}}\right)^{i_{2}} \frac{1}{\left(i_{2} !\right)^{2}} \\
\cdot\left(\frac{\kappa_{3}\left(\kappa_{3}+1\right)}{\Omega_{3}}\right)^{i_{3}} \frac{1}{\left(i_{3} !\right)^{2}} x^{2 i_{1}+1} \\
\cdot \int_{0}^{\infty} d x_{2} \int_{0}^{\infty} d x_{3}\left(1+\frac{x^{2}}{x_{2}^{4} x_{3}^{2}} \frac{\Omega_{2}}{\kappa_{2}+1} \frac{\kappa_{1}+1}{\Omega_{1}}+\frac{x^{2}}{x_{2}^{2} x_{3}^{4}} \frac{\Omega_{3}}{\kappa_{3}+1} \frac{\kappa_{1}+1}{\Omega_{1}}\right)^{1 / 2} . \\
\cdot x_{2}^{-2 i_{1}-1+2 i_{2}+1} x_{3}^{-2 i_{1}-1+2 i_{3}+1} e^{-\frac{\kappa_{1}+1}{\Omega_{1}} \frac{x^{2}}{x_{2}^{2} x_{3}^{2}}-\frac{\kappa_{2}+1}{\Omega_{2}} x_{2}^{2}-\frac{\kappa_{3}+1}{\Omega_{3}} x_{3}^{2}} .
\end{gathered}
$$

The last integral is solved using Laplace approximation theorem for solving the two-fold integrals [43], and presented in [11] through equations (22)-(29).

The Figs. 15 and 16 further more represent normalized LCR of product of three Rician RVs versus this product $x$ with variable Rician factor $\kappa_{i}$ and average power $\Omega_{i}$.

LCR increases with increasing of Rician signal power. The influence of signal power on the LCR is more significant for bigger values of Rician factor $\kappa_{\mathrm{i}}$. LCR increases with increasing of $\Omega_{\mathrm{i}}$ for all signal values.

The impact of signal value on the LCR is larger for bigger values of the signal with different $\Omega_{\mathrm{i}}$. It is important to say that system is with better performance for low LCR.

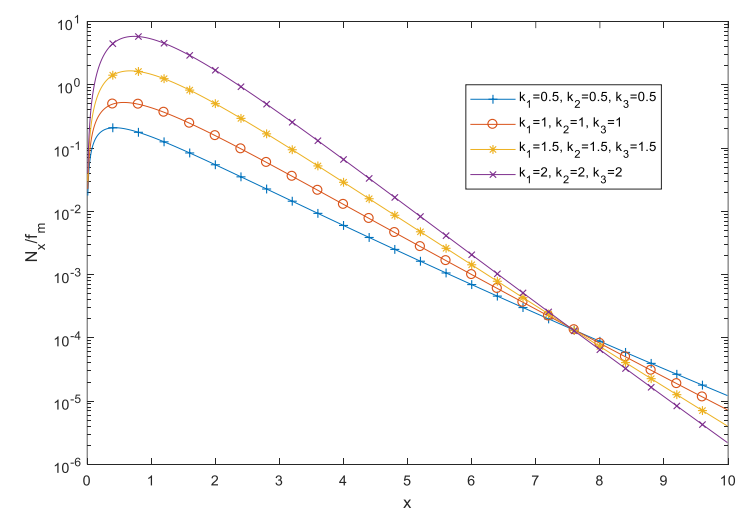

Fig. 15. LCR normalized by $\mathrm{f}_{\mathrm{m}}$ depending on signal envelope $x$ for a few values of Rician factor $\kappa_{\mathrm{i}}$ and signal power $\Omega=1$ [11]. 


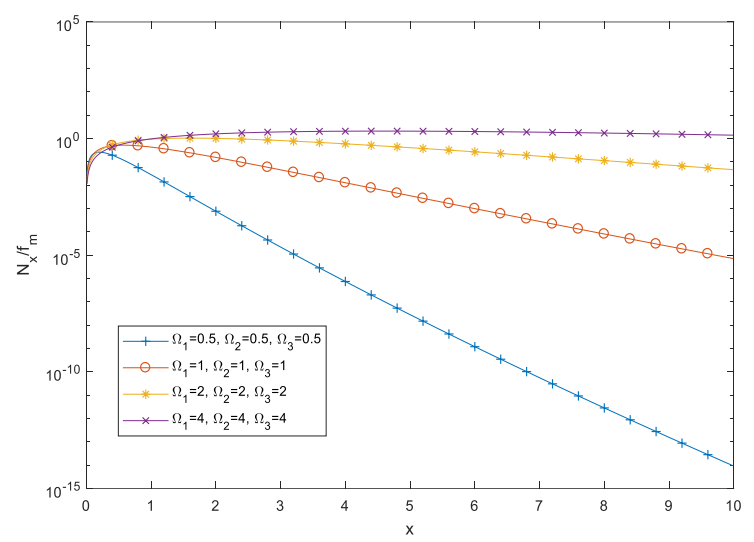

Fig. 16. LCR normalized by $\mathrm{f}_{\mathrm{m}}$ versus signal envelope $x$ for various values of signal powers $\Omega_{\mathrm{i}}[11]$.

\section{B. Average Fade Duration of Product of Three Rician RVs}

AFD informs about duration of staying a power below a given threshold. It is obtained help the LCR [41]. So, AFD is defined by [44, eq. (9)]:

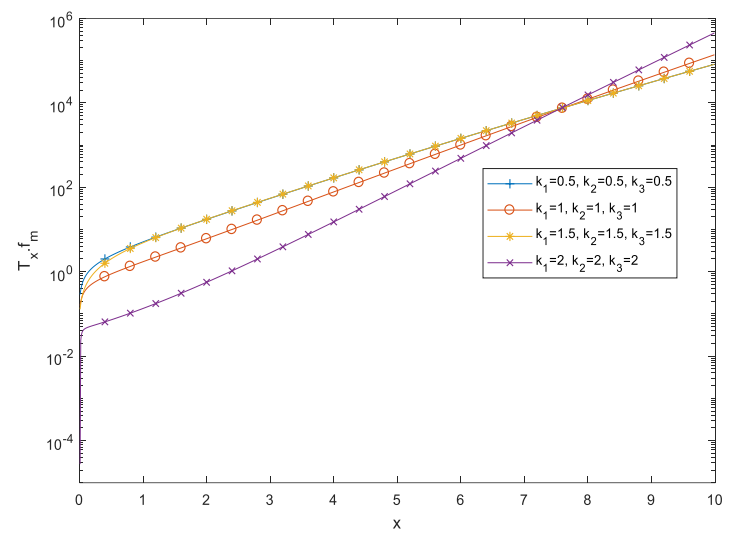

Fig. 17. AFD normalized by $\mathrm{f}_{\mathrm{m}}$ depending on signal envelope $x$ for variable Rician factor $\kappa_{i}$ and signal powers $\Omega_{\mathrm{i}}=1$ [12].

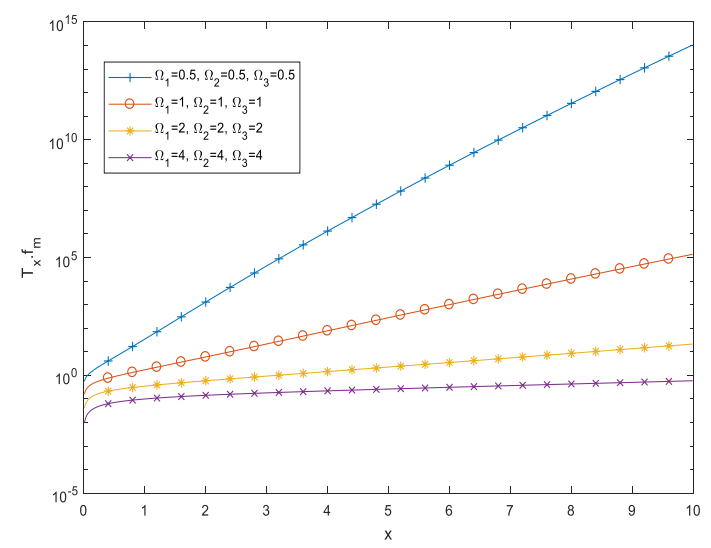

Fig. 18. Normalized AFD versus signal envelope $x$ for $\kappa=1$ and different values of signal powers $\Omega_{\mathrm{i}}[12]$.

$$
T_{x}(x)=\frac{P(x \leq X)}{N_{x}(x)}=\frac{\int_{0}^{X} p_{x}(x) d x}{N_{x}(x)},
$$

where AFD is the ratio of CDF of $x$ given by (2) and solved LCR from (16) [45].

The normalized AFD $\left(T_{x} f_{m}\right)$ of product of three Rician RVs is shown in Figs. 17 and 18 as a function of the envelope $x$. It can be seen from Fig. 17 that AFD has lower values for higher values of $\kappa_{\mathrm{i}}$ and lower envelope $x$. Also, the AFD increases for all signal envelopes and small $\Omega_{\mathrm{i}}$. The impact of $\Omega_{\mathrm{i}}$ is presented in Fig. 18. From this figure is visible that the impact of $\Omega_{\mathrm{i}}$ is bigger for higher $x$.

\section{AN APPLICATION}

Whereas Rician distribution describes fading for radio propagation where the line of sight component and many random weaker signal components are present, Rayleigh distribution models multipath fading where LOS signal component is not present. Because of that, Rayleigh distribution can be analyzed as a special case of general concept of Rician fading. Rayleigh fading becomes from Rician when Rician factor $\kappa$ is zero. Because of that, all analyzed performance of product of three Rician RVs can be used for evaluation the performance of product of three Rayleigh RVs, also for performance of product of two Rayleigh RVs and Rician RV, and CDF of product of two Rician RVs and Rayleigh RV. These results can be used in performance analysis of wireless relay systems with three hops (sections) in the presence of multipath fading:

1) when Rician fading is present in all three hops (sections), and $\kappa_{i} \neq 0, i=1,2,3$,

2) when Rayleigh fading is present in all three hops $\left(\kappa_{1}=\kappa_{2}=\kappa_{3}=0\right)$,

3) when Rayleigh fading is at two hops and Rician at one $\left(\kappa_{1}=\kappa_{2}=0, \kappa_{3} \neq 0\right)$, and

4) when Rayleigh fading is present at one and Rician fading at two hops $\left(\kappa_{1}=0, \kappa_{2} \neq 0, \kappa_{3} \neq 0\right)$.

5) A case with $\kappa \rightarrow \infty$ present the scenario when fading is not present at any hop.

The parameter selection illustrates possible shapes of curves for resulting distribution.

\section{CONCLUSION}

In this article, the expressions for PDF, CDF, outage probability, moments, amount of fading, channel capacity, LCR and AFD of the product of three Rician RVs are performed. This model of RV corresponds to output signal from three-hop relay wireless system mainly in suburban areas, with a pronounced line-of-sight, which is described by Rician distribution. A number of graphics are presented to show the impact of fading parameters. Conventional networks are usually single-hop, with the end devices connected by a direct link, which may cause large path loss. This fact motivates the use of multi-hop topologies to enable energy-efficient connectivity in smart city applications. Because of that, we presented in our papers three-hop cases for different fading distributions. Next 
investigations will be tied to multi-hop environments in the presence of fading described by newer, general distributions, such as: $\alpha-\mu, \kappa-\mu, \eta-\mu, \alpha-\kappa-\mu, \alpha-\eta-\kappa-\mu$, and others.

\section{APPENDIX}

Random variable $x$ is defined as the product of three independent RVs, all with Rician distribution:

$$
x=x_{1} \cdot x_{2} \cdot x_{3} .
$$

Then, based on (A1), $x_{l}$ is:

$$
x_{1}=\frac{x}{x_{2} x_{3}} .
$$

Conditional PDF of $x$ is:

$$
p_{x}\left(x / x_{2} x_{3}\right)=\left|\frac{d x_{1}}{d x}\right| p_{x_{1}}\left(\frac{x}{x_{2} x_{3}}\right),
$$

where:

$$
\frac{d x_{1}}{d x}=\frac{1}{x_{2} x_{3}}
$$

After some substitutions, conditional PDF is:

$$
p_{x}\left(x / x_{2} x_{3}\right)=\frac{1}{x_{2} x_{3}} p_{x_{1}}\left(\frac{x}{x_{2} x_{3}}\right) \text {. }
$$

Then, PDF is obtained by averaging in the form:

$$
\begin{aligned}
& p_{x}(x)= \int_{0}^{\infty} d x_{2} \int_{0}^{\infty} d x_{3} \frac{1}{x_{2} x_{3}} p_{x_{1}}\left(\frac{x}{x_{2} x_{3}}\right) p_{x_{2}}\left(x_{2}\right) p_{x_{3}}\left(x_{3}\right)= \\
&= \frac{2\left(\kappa_{1}+1\right)}{\Omega_{1} \mathrm{e}^{\kappa_{1}}} \sum_{j_{1}=0}^{\infty}\left(\frac{\left(\kappa_{1}+1\right) \kappa_{1}}{\Omega_{1}}\right)^{j_{1}} \frac{1}{\left(j_{1} !\right)^{2}} . \\
& \cdot \frac{2\left(\kappa_{2}+1\right)}{\Omega_{2} \mathrm{e}^{\kappa_{2}}} \sum_{j_{2}=0}^{\infty}\left(\frac{\left(\kappa_{2}+1\right) \kappa_{2}}{\Omega_{2}}\right)^{j_{2}} \frac{1}{\left(j_{2} !\right)^{2}} . \\
& \cdot \frac{2\left(\kappa_{3}+1\right)}{\Omega_{3} \mathrm{e}^{\kappa_{3}}} \sum_{j_{1}=0}^{\infty}\left(\frac{\left(\kappa_{3}+1\right) \kappa_{3}}{\Omega_{3}}\right)^{j_{1}} \frac{1}{\left(j_{3} !\right)^{2}} . \\
& \int_{0}^{\infty} d x_{2} \int_{0}^{\infty} d x_{3} x_{2}^{-1-2 j_{1}-1+2 j_{2}+1} x_{3}^{-1-2 j_{1}-1+2 j_{3}+1} \\
& \cdot x^{2 j_{1}+1} e^{-\frac{\kappa_{1}+1}{\Omega_{1}}\left(\frac{x}{x_{2} x_{3}}\right)^{2}-\frac{\kappa_{2}+1}{\Omega_{2}} x_{2}^{2}-\frac{\kappa_{3}+1}{\Omega_{3}} x_{3}^{2}} .
\end{aligned}
$$

\section{ACKNOWLEDGMENT}

This article is partially worked within the projects of the Serbian Ministry of Education, Science and Technological Development under numbers TR-33035 and III-44006.

\section{REFERENCES}

[1] N. Athanasopoulos, P. Tsiakas, K. Voudouris, I. Georgas, and G Agapiou, "Multi-hop Relay in Next Generation Wireless Broadband
Access Networks: An Overview", International Conference on Mobile Lightweight Wireless Systems 2010, pp 543-554, https://doi.org/10.1007/978-3-642-16644-0_47

[2] M. S. Aslam et al., "Exploring Multi-Hop LoRa for Green Smart Cities," in IEEE Network, vol. 34, no. 2, pp. 225-231, March/April 2020, doi 10.1109/MNET.001.1900269

[3] Z. Hadzi-Velkov, N. Zlatanov, N., and G. K. Karagiannidis, "Level crossing rate and average fade duration of the multihop Rayleigh fading channel", IEEE International Conference on Communications, Beijing, China, 19-23 May 2008, doi: 10.1109/ICC.2008.835

[4] A. D. Cvetkovic, N. M. Sekulovic, D. S. Krstic, E. S. Mekic, Z. J. Popovic, M. C. Stefanovic, " Performance analysis of multi-hop system in Nakagami- $m$ environment", Proc. of the International conference of the Institute for Environment, Engineering, Economics and Applied Mathematics: Circuits, Systems, Signals (CSS), 2010, Sliema, Malta, September 15-17, 2010, pp. 276-279.

[5] A. Panajotović, N. Sekulović, A. Cvetković, and D. Milović, "System performance analysis of cooperative multihop relaying network applying approximation to dual-hop relaying network", International Journal of Communication Systems, e4476, 2020, doi:10.1002/dac.4476

[6] M. Kamal, M. Ibrahim, S., Mir, and M.N. Aman, "Comparison of multihop relaying protocols in cognitive radio networks", 2016 Sixth International Conference on Innovative Computing Technology (INTECH). doi:10.1109/intech.2016.7845084

[7] M. Kamal, T. Yukong, Performance Evaluation of 2-Hop and 3-Hop Relay Network using DF, AF and DAF Relaying Protocols, Blekinge Institute of Technology, Master Thesis, February 2012.

[8] D. Krstic, P. Nikolic, Z. Popovic, M. Stefanovic, "First and second order characteristics of wireless three-hop relay channel with presence of Rayleigh fading", Journal of Telecommunications and Information $\begin{array}{llll}\text { Technology (JTIT), } & \text { no. 2/2020, } & \text { pp. }\end{array}$ https://doi.org/10.26636/jtit.2020.144020

[9] D. Krstic, P. Nikolic, I. Vulic, S. Minic, M. Stefanovic, "Performance of the product of three Nakagami-m random variables", Journal of Communications Software and Systems (JCOMSS), vol.16, no. 2, June 2020, pp. 122-130. doi: http://dx.doi.org/10.24138/jcomss.v16i2.989

[10] D. Krstic, P. Nikolic, Z. Popovic, S. Minic, M. Stefanovic, "Moments of Signals over Wireless Relay Fading Environment with Line-of-Sight", 28th International Conference on Software Telecommunications and Computer Networks - SoftCOM 2020, Hvar, Croatia, September 17-19, 2020. DOI: 10.23919/SoftCOM50211.2020.9238347

[11] D. Krstic, M. Stefanovic, M. M. Bani Yassein, S. Aljawarneh, P. Nikolić, "Statistics of the Product of Three Rician Random Processes with Application", International Conference on Data Science, E-learning and Information Systems 2018, Data'18, Madrid, Spain, October 1-2, 2018. doi: $10.1145 / 3279996.3280015$

[12] D. Krstic, P. Nikolic, S. Minic, Z. Popovic, "Some Performance of Threehop Wireless Relay Channels in the Presence of Rician Fading", The Sixteenth International Conference on Wireless and Mobile Communications ICWMC 2020, October 18, 2020 to October 22, 2020 Porto, Portugal, pp. 18-23.

[13] P. B. Devi, A. Yengkhom, M. I. Hasan, and G. Chandwani, "Capacity of Dual Branch MRC System Over Correlated Nakagami-m Fading Channels-A Review", Wireless Personal Communications, 98(2), pp.1735-1752, 2017. doi:10.1007/s11277-017-4942-4

[14] C. B. Issaid and M.-S. Alouini, "Level Crossing Rate and Average Outage Duration of Free Space Optical Links", IEEE Transactions on Communications, Vol. 67, Issue: 9, Sept. 2019, pp. 6234 - 6242. doi:10.1109/tcomm.2019.2918324

[15] P. M. Shankar, Fading and Shadowing in Wireless Systems, Springer, New York Dordrecht Heidelberg London. 2012, doi: 10.1007/978-14614-0367-8

[16] G. L. Stüber, Principles of Mobile Communications, 2nd ed., MA, USA, Springer, 2001.

[17] M. K. Simon and M. S. Alouini, Digital Communication over Fading channels, (2nd ed.). New York, Wiley-IEEE Press., 2004

[18] N. Bhargav, C. R. Nogueira da Silva, Y. J. Chun, E. J. Leonardo, S. L. Cotton and M. D Yacoub, "On the product of two $\kappa-\mu$ random variables and its application to double and composite fading channels", IEEE Transactions on Wireless Communications, Vol. 17, No. 4, April 2018, pp. 2457-2470.

[19] L. Moreno-Pozas, F. J. Lopez-Martinez, J. F. Paris, and E. Martos-Naya, "The $\kappa-\mu$ Shadowed Fading Model: Unifying the $\kappa-\mu$ and $\eta-\mu$ Distributions", IEEE Transactions on Vehicular Technology, 65(12),pp. 9630-9641, 2016. doi:10.1109/tvt.2016.2525721 
[20] S. Khatalin, "On the channel capacity of SSC diversity system in $\eta-\mu$ and $\kappa-\mu$ fading environments", $A E U$ - International Journal of Electronics and Communications, 69(11), 2015, pp. 16831699. doi:10.1016/j.aeue.2015.08.002

[21] L. Kong, G. Kaddoum, and D. B. da Costa, "Cascaded $\alpha-\mu$ Fading Channels: Reliability and Security Analysis”, IEEE Access, Vol.6, May 2018. doi:10.1109/access.2018.2833423

[22] E. J. Leonardo and M. D. Yacoub, "Statistics of the product of arbitrary $\alpha-\mu$ variates with application", IEEE 25th Annual International Symposium on Personal, Indoor, and Mobile Radio Communication (PIMRC), Washington, DC, USA, 2-5 Sept. 2014, pp. 7376, doi:10.1109/pimrc.2014.7136135

[23] M. Savic, M. Smilic, B. Jakšic, "Analysis of Shannon capacity for SC and MRC diversity systems in $\alpha-\kappa-\mu$ fading channel", University Thought, Publication in Natural Sciences, Vol.8, No.2, 2018. doi: 10.5937/univtho8-19491

[24] J. M. Moualeu, D. B. da Costa, F. J. Lopez-Martinez, and R. A. A. de Souza, "On the Performance of $\alpha-\eta-\kappa-\mu$ Fading Channels", IEEE Communications Letters, Vol. 23, Issue: 6, June 2019, pp. $967-970$. DOI 10.1109/LCOMM.2019.2910526

[25] X. Deng, W. Zhang, and C. Tellambura, "Amount of Fading Analysis for Transmit Antenna Selection in MIMO Systems", 2007 IEEE Wireless Communications and Networking Conference, WCNC 2007, pp. 11621166. doi:10.1109/wcnc.2007.220

[26] T. Taniguchi, Y. Karasawa, and M. Tsuruta, "An analysis method of double fading MIMO channels including LOS environments", 2008 IEEE 19th International Symposium on Personal, Indoor and Mobile Radio Communications, doi:10.1109/pimrc.2008.4699512

[27] M. Stefanović, S. Panić, J. Anastasov, A. Cvetković, Z. Popović, "Capacity Evaluation for Maximal Ratio Combining over $\kappa-\mu$ Fading Channels", Serbian Journal of Electrical Engineering, Vol. 8, No. 2, May 2011, pp. 221-228.

[28] M. M. Smilić, B. S. Jakšić, D. N. Milić, S. R. Panić, P. Ć. Spalević, "Channel Capacity of the Macrodiversity SC System in the Presence of kappa-mu Fading and Correlated Slow Gamma Fading", Facta Universitatis, Series: Electronics and Energetics, Vol. 31, No 3, September 2018, pp. 447-460. https://doi.org/10.2298/FUEE1803447S

[29] M. C. Stefanovic, D. S. Krstic, B. Z. Milosevic, J. A. Anastasov, S. R Panic, "Channel capacity of maximal-ratio combining over correlated Nakagami-m fading channels", 9th International Conference on Telecommunication in Modern Satellite, Cable, and Broadcasting Services TELSIKS, 7-9 Oct. 2009, Nis, Serbia, DOI 10.1109/TELSKS.2009.5339434

[30] G. Pandeeswari, M. Suganthi, and R. Asokan, Performance of single hop and multi hop relaying protocols in cognitive radio networks over Weibull fading channel. Journal of Ambient Intelligence and Humanized Computing. 2020, doi:10.1007/s12652-020-01739-z

[31] N. A. Androutsos, H. E. Nistazakis, H. G. Sandalidis, E. Leitgeb, G. S Tombras, "A dual-hop equivalent structure of a generalised multi-hop free-space optics network", IET Communications, Vol.15, Issue5, March 2021, Pages 730-735, https://doi.org/10.1049/cmu2.12115

[32] M. T. Mamaghani, A. Kuhestani, and H. Behroozi, "Can a multi-hop link relying on untrusted amplify-and-forward relays render security?", Wireless Networks, doi:10.1007/s11276-020-02487-w

[33] A. Doukas. "Rician K Factor Estimation for Wireless Communication Systems", 2006 International Conference on Wireless and Mobile Communications. doi:10.1109/iccgi.2006.81

[34] S. O. Rice, "Mathematical analysis of random noise", Bell System Technical Journal, 23(3), 1944, pp. 282-332. doi:10.1002/j.15387305.1944.tb00874.x

[35] N. Shroff and K. Giridhar, "Biased estimation of Rician K factor", 6th International Conference on Information, Communications \& Signal Processing, 2007, doi:10.1109/icics.2007.4449770

[36] I. Trigui, A. Laourine, S. Affes, and A. Stephenne, "Outage analysis of wireless systems over composite fading/shadowing channels with cochannel interference", IEEE Wireless Communications and Networking Conference, 5-8 April 2009, Budapest, Hungary

[37] H. H. Nguyen and E. Shwedyk, Probability Theory, Random Variables and Random Processes. A First Course in Digital Communications, Cambridge University Press, 2009, pp. 77-134. doi:10.1017/cbo9780511841583.004

[38] B. Holter and G. E. Oien, "On the amount of fading in MIMO diversity systems", IEEE Transactions on Wireless Communications, 4(5), 2498 2507. doi:10.1109/twc.2005.853832a
[39] R. Priyadarshi, M. P. Singh, A. Bhardwaj, and P. Sharma, "Amount of fading analysis for composite fading channel using holtzman approximation", 2017 Fourth International Conference on Image Information Processing (ICIIP). doi:10.1109/iciip.2017.8313759

[40] M. Ibnkahla, Signal Processing for Mobile Communications Handbook, 2005. p. 872.

[41] A. Goldsmith, Wireless Communications, Cambridge University Press, 2005, DOI: https://doi.org/10.1017/CBO9780511841224

[42] W. C. Jakes, Microwave Mobile Communications. Piscataway, NJ: IEEE Press, 1994.

[43] J. L. Lopez and P. J. Pagola, "A simplification of the laplace method for double integrals. Application to the second appell function", Electronic Transactions on Numerical Analysis, vol. 30, 2008.

[44] T. T. Tjhung and C. C. Chai, "Fade statistics in Nakagami-lognormal channels", IEEE Transactions on Communications, 1999, 47(12), pp. 1769-1772. doi:10.1109/26.809692

[45] X. Dong and N. C. Beaulieu, "Average level crossing rate and average fade duration of selection diversity", IEEE Communications Letters, 5(10), 2001, pp. 396-398, doi:10.1109/4234.957373

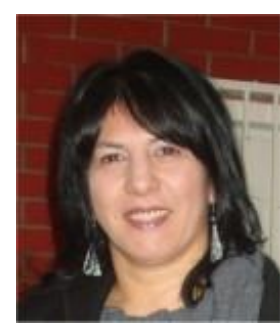

Dragana S. Krstic was born in Pirot, Serbia. She received the B.Sc, M.Sc. and PhD. degrees in Electrical Engineering from Faculty of Electronic Engineering, University of Nis, Serbia in 1990 1998. and 2006, respectively. She is working at Faculty of Electronic Engineering, University of Nis, since 1990. Her field of interest includes telecommunications theory, optical, wireless, mobile and satellite telecommunication systems. As author/co-author, she wrote about 290 scientific research papers, of which about 65 are printed in international journals, several in national journals, near 160 are referred at international symposia and conferences and around 30 at national. Dr Krstic had 32 plenary and keynote lectures, panels and tutorials by invitation at international conferences and some faculties. She is also the member of editorial boards of a few international journals and reviewer for many of them. Also, she is a member of the technical program committees for 145 conferences and reviewer for about new 150 ones.

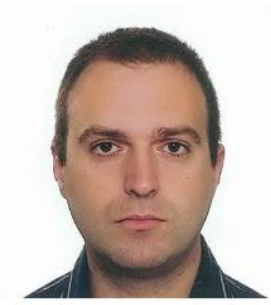

Petar B. Nikolic was born in Pirot, Serbia, in 1974. He graduated at the Faculty of Electronic Engineering, University of Nis, Serbia and received M.Sc. and Ph.D. degrees in 2008 and 2016 , respectively. He is working in the company Tigar Tyres, Pirot. The main research interest is connected with the wireless communication systems. He has written or co-authored a considerable number of papers, published in eminent journals and conferences proceedings.

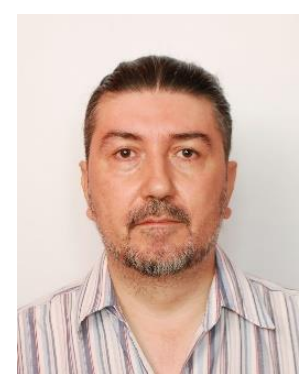

Zoran J. Popovic was born in Cacak, Serbia. He graduated at the Faculty of Electronic Engineering, University of Nis, Serbia and received M.Sc. and PhD. degrees in 2003 and 2011, respectively. His field of interest includes telecommunications theory, mobile and wireless communication systems. He has written or coauthored a considerable number of papers published in eminent journals and conferences proceedings. Now, Dr Popovic is working as professor in Higher Technical Professional School in Zvecan, Department of information and communications technologies. 


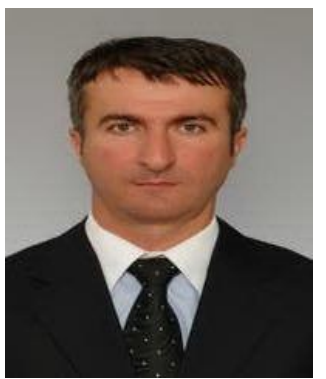

Siniša G. Minić was born in Gulija, Leposavic Serbia. He received the B. Sc and M. Sc. degrees in electrical engineering from the Faculty of Electronic Engineering, in 1996 and 2001, respectively and $\mathrm{PhD}$. degree from Technical Faculty in Čačak, Serbia, in 2004. He is full professor at the Department of Informatics and Mathematics, Faculty of Teacher Education in Prizren - Leposavic University of Pristina Kosovska Mitrovica, Serbia, where he lectures: Computers in education, Information Technology, Educational technology, Informatics in pedagogy, Information technology in teaching. Areas of interest include telecommunications, information technology, information technology in education. Prof. Minic has authored about 60 scientific peer-reviewed papers and a large number of projects and patents. He is a member of the technical program committees and reviewer for several international journals and symposia.

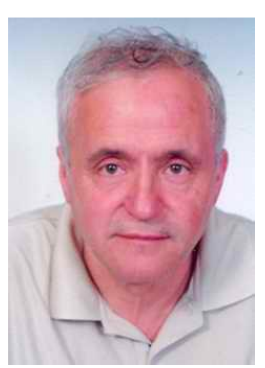

Mihajlo C. Stefanović received the B. Sc., M. Sc. and $\mathrm{PhD}$ degrees in electrical engineering from the Faculty of Electronic Engineering, Department of Telecommunications, University of Nis, Serbia, in 1971, 1976 and 1979, respectively. His primary research interests are statistical communication theory, optical and wireless communications.

He has written or coauthored a great number of journal publications. He has written five monographs, too. He was a mentor to hundreds of graduates, for dozens of master's theses and doctoral dissertations, and many times a member of commissions for the defense of such works. Now, Dr. Stefanović is a retired professor of the Faculty of Electronic Engineering, University of Nis. 